\title{
Difficulties in diagnosing and treating depression in the terminally ill cancer patient
}

\author{
Mari Lloyd-Williams
}

\begin{abstract}
It is estimated that for a quarter of all patients with advanced metastatic cancer, depression will be significant symptom. However up to $80 \%$ of the psychological and psychiatric morbidity which develops in cancer patients goes unrecognised and untreated. One of the main difficulties in establishing a diagnosis of an illness where there are no biological markers, physical signs, or diagnostics tests is deciding what can be called "appropriate sadness" as patients approach the end of life and what is a depressive illness.

Criteria for diagnosing depression are discussed together with reasons why established screening tools used in other populations may not be appropriate to screen for depression in terminally ill patients. The use of antidepressants and the role of cognitive therapies are also discussed. For effective treatment of a depressive illness, treatment with antidepressant medication needs to be initiated sooner rather than later and patients may need close supervision to ensure compliance. The awareness of depression, the identification of symptoms, and the initiation of treatment is essential if patients are to be offered optimum palliation of psychological as well as physical symptoms.

(Postgrad Med f 2000;76:555-558)
\end{abstract}

Keywords: depression; terminal illness; screening

Over the last 30 years, there have been major improvements in the care of terminally ill cancer patients - this is due largely to the development of the hospice movement and greater education on the control of pain and other symptoms. It is estimated that for a quarter of all patients with advanced metastatic cancer, depression will be a significant symptom, ${ }^{1}$ however up to $80 \%$ of the psychological and psychiatric morbidity that develops in cancer patients goes unrecognised and untreated. ${ }^{2}$ The exact aetiology of depression in cancer and terminal illness is unknown, but several theories have been put forward. Greer and Silberfarb suggested that the emotional impact of a cancer diagnosis, side effects of treatment, progression of cancer with associated disability, and symptoms and cerebral dysfunction associated with carcinomatosis were important precipitating factors. ${ }^{3}$ The presence of excellent communication skills by medical and nursing staff and the ability to allow patients to express their thoughts and fears are all vitally important as patients often conceal their feelings in order to protect staff. ${ }^{4}$ Depression is an important symptom that has great impact on quality of life for patients with advanced metastatic cancer, but its diagnosis and appropriate management is difficult.

One of the main difficulties in establishing a diagnosis of an illness where there are no biological markers, physical signs, or diagnostic tests is deciding what can be called "appropriate sadness" as patients approach the end of life and what is a depressive illness.

\section{Assessment of depression}

The diagnosis of depression is by a clinical psychiatric interview. A history of the patient is taken looking at present events and past experiences which may explain the current behaviour or symptoms. The childhood history, developmental milestones, academic and personal development, and premorbid personality are included in the history. The second part of the interview comprises the mental state examination when the patient's observable behaviour and symptoms, for example auditory hallucinations, are documented. Patients are asked about their thoughts regarding the future, the presence of tearfulness, guilt regarding past events, and to rate their own mood. They are also asked if they have any suicidal ideation.

Depression is diagnosed if patients have a persistent low mood and at least four of the following symptoms which are present most of the day for the preceding two weeks. ${ }^{5}$

(1) Diminished interest or pleasure in all or almost all activities.

(2) Psychomotor retardation or agitation.

(3) Feelings of worthlessness or excessive and inappropriate guilt.

(4) Diminished ability to concentrate and think.

(5) Recurrent thoughts of death and suicide.

(6) Fatigue and loss of energy.

(7) Significant weight loss or gain.

(8) Insomnia or hypersomnia.

In patients with advanced cancer where symptoms 6-8 are present, strategies for the assessment and management of depression in patients with advanced cancer have been proposed which include ${ }^{6}$ :

(1) Thorough physical assessment and relief of poorly controlled symptoms.

(2) Underlying organic factors detected and treated where possible.

(3) Normal sadness and grief at the end of life differentiated from those indicating a depressive disorder.

(4) Supportive psychotherapy for the patients to reduce sense of isolation.

(5) Family intervention to support relatives.
Submitted 26 January 1999 Accepted 7 December 1999 
(6) Use of selected antidepressants.

Although these guidelines may be helpful, they do not detail how to differentiate between the sadness and grief at the end of life and a depressive disorder. There is also the complex problem of deciding which symptoms, especially the somatic symptoms of depression, for example sleep or appetite disturbance, may be attributable to the cancer and which may be due to depression. Endicott proposed that in the assessment of depression in the patient with cancer, somatic symptoms should be substituted as follows ${ }^{7}$ :

- Poor appetite/weight gain; substituted for fearfulness or depressed appearance in body or face.

- Insomnia/hypersomnia; substituted for social withdrawal or decreased talkativeness.

- Loss of energy/fatigue; substituted for brooding, self pity, pessimism.

- Diminished concentration or slowed thinking; substituted for cannot be cheered up, does not smile, no response to good news or funny situations.

Endicott also stressed the importance of asking patients with cancer about suicidal ideation.

In an attempt to improve the detection of depression in patients with advanced cancer, screening tools have been widely used. The majority of such tools contain a number of symptoms or feelings and the patient indicates their own response and the scores are calculated by the person administering the scale. Assessment tools are not diagnostic tools and can only serve to indicate whether a patient has particular psychiatric symptoms suggestive of a diagnosis of depression after which a decision is taken whether to treat for depression or to refer the patient for further assessment.

One of the most widely used instruments is the hospital anxiety and depression (HAD) scale; it is a 14 item scale devised by Zigmond and Snaith for use with medical patients. ${ }^{8}$ The depression subscale is based on anhedoniathe complete loss of enjoyment; the concept of anhedonia has been described as the "exclusion from the pleasure dome". ${ }^{9}$ Ibbotson et al found that the HAD scale performed best in patients with cancer who were disease free or receiving active treatment, ${ }^{10}$ and although widely used, it may not be robust enough for routine screening in terminally ill patients.

\section{Psychotropic medication and choice of} antidepressant

Maguire et al state that the principles of treatment with antidepressant medication in cancer patients should be the same as in any other patient. ${ }^{11}{ }^{12}$ However, antidepressants are only prescribed for a minority of terminally ill patients who may benefit from them and in the vast majority of cases at a very late stage in the patients illness when there is insufficient time for any therapeutic effect. ${ }^{13}$

There are several misconceptions regarding the prescribing of antidepressant drugs. These include terminally ill patients not meriting psychotropic medication as they have an obvious reason for being depressed; the belief that psychological treatments are better or that drugs are not compatible with such therapies; and unfounded concern over addiction and dependence.

In a study of 146 patients with cancer referred to a liaison psychiatry service antidepressants were prescribed for $67 \%$ of patients with $80 \%$ showing a good clinical response to treatment with few side effects. ${ }^{14}$

The importance of prescribing an adequate dosage of medication for an adequate length of time can not be overemphasised. ${ }^{15}$

As the number of psychotropic drug increase, the choice of antidepressant becomes wider. There are two main classes of antidepressants commonly prescribed: the tricyclics and the selective serotonin reuptake inhibitors (SSRIs); both appear to be equally efficacious when used in adequate dosages. One of the main disadvantages of tricyclics is the anticholinergic side effects-for example dry mouth and constipation-symptoms which are already present in many terminally ill patients because of their advanced cancer. Tricyclics are contraindicated in patients with cardiac conduction disease and may cause postural hypotension especially if patients are dehydrated and are toxic in overdose. However they have beneficial side effects of weight gain which is advantageous for many terminally ill patients and their sedating effect can be exploited by administering the drug at night.

The SSRIs were first developed in the 1980s. They do not have the anticholinergic, cardiac, or sedative effects of the tricyclic drugs, but may cause gastrointestinal disturbance, nausea and diarrhoea, but are safer in overdose. Concern has been expressed recently about the increased risk of gastrointestinal intestinal bleeding and the use of SSRIs. ${ }^{16}$ Most antidepressants take two to three weeks before an effect on mood is observed, however sedative and antianxiety effects are observed earlier.

Compliance with medication can be a problem and patients started on antidepressants may need close monitoring and encouragement to continue with medication by specialist nurses or medical staff during the early weeks of treatment. Psychostimulants such as dexamphetamine give patients a sense of overall wellbeing, increase appetite and elevate mood. They work by promoting the release of biogenic amines and are administered early in the day to avoid insomnia. The dose is normally maintained for about seven to 10 days and slowly reduced. They are generally safe, but care needs to be taken in patients with multiple organ failure. Burns and Eisendrath reported the beneficial effects of dextroamphetamine as a treatment for depression in the terminally ill. ${ }^{17}$ They may have a role in treating depression during terminal illness due to the early therapeutic effect after administration, and although prescribed frequently in the United States, are rarely used in the UK.

The choice of antidepressant medication is therefore governed by which drug may be best suited to each individual patient. The selective serotonin reuptake inhibitors appear to be less 


\section{Key points}

- Lack of awareness of depression in patients with advanced metastatic cancer.

- Difficulty in distinguishing between sadness and depression leads to large number of patients not being treated.

- Antidepressants are prescribed at low doses and are started too late for a therapeutic effect to be obtained.

toxic and better tolerated. A large study of antidepressant use in the community found that fewer patients were discontinued on the SSRIs compared with tricyclic antidepressants and that the former appeared to be better tolerated and therefore compliance was improved. ${ }^{18}$

\section{Non-pharmacological interventions}

Although patients who are suffering from depression require psychotropic medication, psychological support can enhance well being and promote recovery. Counselling is widely available, but to date there is very little evidence to suggest that counselling alone is beneficial for patients who are depressed. One study by Linn et al did show evidence of benefit in terms of depression in men with terminal cancer, ${ }^{19}$ but the counsellors had a very high level of involvement visiting patients several times weekly and frequently being present at the time of death. Many patients with terminal cancer feel isolated by their illness and derive benefit from meeting other patients in a similar situation. Hospices often have attached day centres to give patients the opportunity to meet others in a social environment.

Psychotherapy has also been used in patients with cancer, and most widely in the United States by Spiegel who identified four basic components of psychotherapy when used in patients with cancer, that is social support especially when group psychotherapy is used, emotional expression, cognitive restructuring and coping skills training. ${ }^{20}$ There have been studies suggesting that medically ill patients and cancer patients who develop depression have a different pattern of coping skills to other patients. ${ }^{21}$ Adjuvant psychological therapy has been found to be beneficial in patients with cancer, although studies to date have not found a significant effect on depression. The theoretical framework is devised from the premise that cancer related psychological distress stems from the personal meaning of the disease to the patient and the patient's own coping skills. ${ }^{22}$ Patients were seen for six one hour sessions and the main components are to induce a fighting spirit, encourage expression of emotions, to challenge negative behaviour, and to look at cognitive techniques which may help negative behaviours.

\section{Conclusions}

Apart from the distress that a depressive illness causes in terms of quality of life for terminally
Key features important in depression in the terminally ill

- Guilt

- Feelings of worthlessness and helplessness

- Social withdrawal

- Suicidal ideation

\section{Treatment of depression}

- Early initiation of treatment.

- Adequate doses for adequate period of time

- Tricyclics and SSRIs have similar efficacy but SSRIs may be better tolerated therefore increase compliance.

- Psychological treatments, for example cognitive therapy and adjuvant psychological therapy, may be helpful in addition to antidepressants.

- Psychostimulants are known to work rapidly but are not commonly used by UK palliative care teams.

\section{Questions (answers at end of paper)}

(1) What percentage of terminally ill patients are thought to have symptoms of depression?

(2) What are the main symptoms which can aid the diagnosis of depression in the terminally ill patient?

(3) Are rating scales useful for diagnosing depression in the terminally ill?

(4) Which antidepressants could be prescribed for terminally ill patients?

(5) Which other class of drugs may be useful in treating depression at the end of life?

ill patients it can also lead to poor pain control and difficulty in the control of other symptoms. There is a consensus in the mental health field that there is under recognition and too much diversity in the treatment of depression within different mental health settings. ${ }^{23}{ }^{24}$ House states that the majority of physically ill patients with mood disorder are cared for by those with little or no psychiatric training and methods of improving their clinical skills in this area should be explored..$^{25}$

The diagnosis of depression in terminally ill patients is a challenge and it is important that the prevalence of depression and available therapies are highlighted. ${ }^{26}$ Once patients are identified as being depressed, they require adequate treatment which will frequently involve antidepressant medication. For effective treatment of a depressive illness, treatment needs to be initiated sooner rather than later and patients may need close supervision to ensure compliance. The awareness of depres- 
sion, the identification of symptoms and the initiation of treatment by all who care for and

\section{Answers}

(1) It is believed that one in four terminally ill patients have symptoms of depression but that up to $80 \%$ of this depression may not be recognised and therefore untreated.

(2) The main symptoms which should be elicited when assessing a terminally ill patients for depression are guilt, worthlessness, hopelessness, social withdrawal, expressionless features, and thoughts of self harm or suicide.

(3) Rating scales with proved validity can be useful as a screening tool if they have predefined cut off thresholds. Many instruments used in other settings are not appropriate for terminally ill patients because of their content or the over reliance on somatic symptoms.

(4) The principles of prescribing for terminally ill patients should be as for any other patients. The efficacy of antidepressant drugs appear to be the same as for non-cancer patients. The side effects of the tricyclic drugs, for example dry mouth, may make compliance a problem. The SSRIs may cause gastrointestinal side effects but generally appear to be better tolerated. However all patients require close supervision and encouragement to comply with antidepressant medication.

(5) Psychostimulants are used extensively in the United States as mood elevators and have the advantage of showing a rapid improvement-they are, however, rarely used by palliative care teams in the UK, but research on their use is being carried out. treat terminally patients is essential if patients are to be offered optimum symptom control of psychological as well as physical symptoms.

1 Barraclough J. Cancer and emotion. Chichester: Wiley, 1994.

2 Maguire P. Improving the detection of psychiatric problem in cancer patients. Soc Sci Med 1985;20:819-23.

3 Greer S, Silberfarb P. Psychological concomitants of cancer current state of research. Psychol Med 1982;12:563-73.

4 Maguire P, Faulkner A, Booth K, et al. Helping cancer patients disclose their concerns. Eur F Cancer 1996;32A:7881 .

5 American Psychiatric Association. Diagnostic and statistical manual of mental disorders. 4th Ed. Washington, DC: American Psychiatric Association, 1994.

6 Haig R. Management of depression in patients with advanced cancer. Med f A ust 1992;156:499-503.

7 Endicott J. Measurement of depression in patients with cancer. Cancer 1984;53:2243-8.

8 Zigmond AS, Snaith RP. The hospital anxiety and depression scale. Acta Psychiatr Scand 1983;67:361-70.

Snaith P. Anhedonia: exclusion from the pleasure dome. BMF 1992;305:134.

10 Ibbotson T, Maguire P, Selby T, et al. Screening for anxiety and depression in cancer patients: the effects of disease and treatment. Eur f Cancer 1993;30A:37-40.

11 Maguire P. Psychological morbidity associated with cancer and cancer treatment. Clinics in Oncology 1985;4:559-75.

12 Maguire P, Hopwood P, Tarrier N, et al. Treatment of depression in cancer patients. Acta Psychiatr Scand 1985; 72(suppl 320):81-4.

13 Lloyd-Williams M, Friedman T, Rudd N. A survey of antidepressant prescribing in hospices. Palliat Med 1999;13: 243-8.

14 Chaturvedi S, Maguire P, Hopwood P. Antidepressant medications in cancer patients. Psycho-Oncology 1994;3:5760.

15 Massie M, Holland J. Diagnosis and treatment of depression in the cancer patient. $\mathcal{F}$ Clin Psychiatry 1984;3:25-8.

16 de Abajo F, Garcia L, Rodriguex G, et al. Assocation between selcective serotonin reuptake inhibitors and upper gastrointestinal bleeding: population based case control group. BMF 1999;319:1106-9.

17 Burns M, Eisendrath S. Dextroamphetamine treatment for depression in terminally ill patients. Psychosomatics 1992;35: $80-4$.

18 Martin R, Hilton S, Kerry S, et al. General practitioner's perceptions of the tolerability of antidepressant drugs: a comparison of selective serotonin inhibitors and tricyclic antidepressants. BMF 1997;314:646-51.

19 Linn M, Linn B, Harris R. Effects of counselling for late stage cancer patients. Cancer 1982;49:1048-55.

20 Spiegel D. Cancer and depression. $\mathrm{Br} \quad \mathcal{F}$ Psychiatry 1996;168:109-16.

21 Parle M, Jones B, Magure P. Maladaptive coping and affective disorders among cancer patients. Psychol Med 1996;26: $735-44$.

22 Greer S, Moorey S. Adjuvant psychological therapy for cancer patients. Palliat Med 1997;11:240-4.

23 Brugha T. Depression undertreatment: lost cohorts, lost opportunities? Psychol Med 1995;25:3-6.

24 Geddes J, Reynolds S, Streiner S, et al. Evidence based practice in mental health. Evidence-Based Mental Health 1998;1:4-5.

25 House A. Mood disorders in the physically ill-problems of definition and measurement. F Psychosom Res 1988;32:34553.

26 Lynch $M$. The assessment and prevalence of affective disorders in advanced cancer. F Palliat Care 1995;11:10-18. 